Images du travail, travail des images

$5 \mid 2018$

Le travail à l'écran : mise en scène des groupes professionnels par les médias

\title{
Dedans, dehors : la place de l'instrumentiste- instrument en orchestre symphonique
}

Inside, outside: the place of the symphonic orchestra's instrumentalistinstrument

\section{Samuel Chagnard}

\section{(2)enEdition}

\section{Journals}

Édition électronique

URL : http://journals.openedition.org/itti/846

DOI : $10.4000 /$ itti.846

Éditeur

Université de Poitiers

Référence électronique

Samuel Chagnard, «Dedans, dehors : la place de l'instrumentiste-instrument en orchestre

symphonique », Images du travail, travail des images [En ligne], 5 | 2018, mis en ligne le 01 février 2018 consulté le 14 avril 2021. URL : http://journals.openedition.org/itti/846 ; DOI : https://doi.org/10.4000/ itti.846

Ce document a été généré automatiquement le 14 avril 2021

Images du travail, travail des images 


\section{Dedans, dehors : la place de l'instrumentiste-instrument en orchestre symphonique}

Inside, outside : the place of the symphonic orchestra's instrumentalist-

instrument

Samuel Chagnard

1 L'Orchestre de Paris propose sur son site internet une rubrique « Figures de notes ${ }^{1}$ » où des musicien.ne's de l'orchestre présentent leur instrument sous forme de vidéos de dix minutes environ. Cette mise en scène audiovisuelle montre une facette du métier de musicien'ne d'orchestre rarement médiatisée. Le métier de musicien.ne d'orchestre symphonique a fait l'objet de plusieurs études au cours des vingt-cinq dernières années (Dupuis, 1993 ; Willener, 1997 ; Lehmann, 2005 ; Coulangeon, 2004 ; Adenot, 2008 ; Ravet, 2011). En étudiant « l'envers de l'harmonie » (Lehmann, 1995), elles ont mis en évidence une multiplicité de hiérarchie à l'origine de tensions entre instrumentistes au sein de l'orchestre pouvant conduire au "désenchantement» (Adenot, 2008) de ce métier. "Figures de notes ", en mettant des musicien'ne's avec leur instrument en situation de représentation (Goffman, 1973) différente du concert, constitue un matériau iconographique particulier pour saisir les conceptions de ce métier. En effet, au-delà de la stricte présentation d'un instrument, et au-delà de l'illustration de la fonction de médiation de l'instrumentiste ${ }^{2}$, cette mise en scène permet de voir le rapport qu'entretiennent les instrumentistes avec leur instrument. Par-delà la description $a$ priori uniquement historique, technique et musicale de leur instrument, chaque instrumentiste propose également une description sociale de son environnement musical, donnant par là à voir le rapport à la musique construit dans son parcours, en particulier le rapport à la division sociale et musicale du travail en orchestre (Lehmann, 2005).

2 L'analyse $^{3} \mathrm{du}$ dispositif audiovisuel de présentation des instruments permet de rendre compte dans un premier temps des processus conjoints de personnification de l'instrument et d'identification à l'instrument qui transforment l'instrumentiste en 
« instrumentiste-instrument ». Ensuite, je décris comment le dispositif signale la place de chaque instrumentiste-instrument à travers un placement spatial, un rôle musical et un statut social spécifiques et hiérarchisés. Enfin, il s'agit ici de montrer comment le discours des instrumentistes, dans la situation de représentation induite par ce dispositif, alterne entre l'affirmation et l'appréciation de leur place dans l'orchestre et l'aspiration à une place en dehors de celui-ci, et comment cette tension peut s'expliquer par la figure de l'instrumentiste-instrument.

\section{Confondre l'instrumentiste et l'instrument}

«La première harpe solo de l'Opéra de Paris, Emmanuel Ceysson, intègre le Metropolitan Opera de New York. $»^{4}$

3 En mars 2015, la mise en ligne de la rubrique «Figure de notes » sur le site internet de l'Orchestre de Paris fait suite au déménagement de l'orchestre de la Salle Pleyel (8ème arrt) vers la nouvelle salle de concert de la Philharmonie (19ème arrt), inaugurée en janvier 2015. Le responsable du site internet indique que le changement de "localisation implique aussi un changement de public, de catégorie [...], public moins mélomane, moins connaisseur ». Cette nouvelle rubrique s'adresse, pour lui, à ce "public adulte, mélomane sans plus, ou carrément néophyte, pour comprendre un petit peu de quoi est fait un orchestre ». Pour cela, "Figure de notes" propose de "[découvrir] les musiciens et les instruments de l'Orchestre de Paris » (programme de la saison 2015-16, p. 47) avec deux dispositifs : un ensemble de 17 vidéos ${ }^{5}$ d'une dizaine de minutes où des musicien'ne's de l'orchestre présentent leur instrument et un jeu interactif $^{6}$ de questions pour tester ses connaissances sur les instruments.

Comprendre «de quoi est fait un orchestre " implique ainsi la présentation des instruments de l'orchestre, mais également des instrumentistes qui les jouent afin de «mettre en avant ces personnalités, parce qu'on a trop souvent l'impression qu'un orchestre c'est un ensemble de musiciens indifférenciés, alors que chez nous, 119 musiciens, c'est 119 individualités » (Responsable du site internet).

\subsection{L'instrumentiste-instrument}

5 La préoccupation de distinguer l'instrument et son instrumentiste constitue le principe même du scénario commun à chacune des 17 vidéos. La séquence introductive (entre 30 et 60 secondes) comporte un extrait du «Boléro» de Maurice Ravel joué par l'instrumentiste et une présentation générale de l'instrumentiste puis de l'instrument. La vidéo est ensuite séquencée en six rubriques : les trois premières insistent sur la singularité de l'instrument («mode d'emploi de l'instrument », « les différents sons de l'instrument ", "l'instrument vu par les compositeurs ») et les trois dernières mettent l'accent sur la singularité de l'instrumentiste liée à cet instrument («pourquoi j'ai choisi l'instrument ", "mon solo préféré ", « la question qu'on me pose toujours sur mon instrument »). Cette organisation a pour but de rendre visible les membres d'un ensemble dans lequel ces "individualités, autant au niveau humain qu'au niveau des instruments, sont peu perçues » (Responsable du site internet, souligné par moi).

6 En cherchant à distinguer chaque instrumentiste avec son instrument parmi l'ensemble des autres membres de l'orchestre, le dispositif vidéo contribue à confondre 
l'instrument et l'instrumentiste en fabriquant une seule et même individualité. Je propose d'appeler « instrumentiste-instrument » cette nouvelle individualité.

7 L'analyse de la séquence introductive donne un aperçu de la confusion entre l'instrumentiste et son instrument. L'extrait monté qui suit présente l'introduction de la vidéo du violoncelliste suivie d'un montage ${ }^{7}$ des titres des 6 rubriques qui structurent chaque vidéo :

Ce média ne peut être affiché ici. Veuillez vous reporter à l'édition en ligne http:// journals.openedition.org/itti/846

- Séquence 1 (0'00-0'17) : La coïncidence de l'inscription du nom de l'instrument avec l'arrivée du mouvement de caméra sur le visage de l'instrumentiste fait associer le nom de l'instrument avec le visage (la «figure ») de l'instrumentiste.

- Séquence 2 (0'18-0'23): L'instrumentiste précise son métier général (musicien-ne) et l'instrument qu'il-elle joue. Les inscriptions présentent le métier spécifique lié à l'instrument : l'instrumentiste.

- Séquence $3\left(0^{\prime} 24-0^{\prime} 45\right)$ : Le vocabulaire employé dans les inscriptions présente l'instrument comme un être humain: une première description de l'instrument de type « anthropométrique » (« date de naissance », « taille », « poids ») proche d'un faire-part de naissance, puis une seconde description de type «identitaire " qui situe "socialement» l'instrument dans l'orchestre (« famille », « parents », « voisins »).

Les associations successives suggérées par les trois séquences - individu-instrument, musicien-ne-instrumentiste et instrument-être humain - confondent au démarrage de chaque vidéo l'individu qui joue et l'instrument joué, fabriquant ainsi l'instrumentisteinstrument.

La notion d'instrumentiste-instrument permet de rendre compte du double mouvement d'assimilation de l'instrumentiste à l'instrument et d'incorporation de l'instrument par l'instrumentiste, et de comprendre ensuite la place qui lui est attribuée.

\subsection{L'humanisation de l'instrument}

11 La constitution de l'instrumentiste-instrument se fait d'abord par la personnification de l'instrument en lui donnant des attributs humains. Les musicien'ne's filmé'e-s humanisent de plusieurs façons l'instrument dont elles-ils jouent. En premier par l'utilisation du vocabulaire de la lutherie proche de celui d'un être vivant, de manière physiologique (le «corps", la «tête", la «peau», la "patte», les "ouïes », la " cheville », le « bec ») ou spirituelle (l'« âme »). Le vocabulaire en référence à la famille souligne ensuite le lien de proximité entre des instruments, ou l'origine de l'instrument (la flûte «sœur » du piccolo, le violoncelle «grand frère » du violon, le tuba «grandpère de la famille » des cuivres ou encore « Mozart, père de la clarinette »).

Dans certaines descriptions, le premier professeur de l'instrumentiste et l'instrument sont associés, voire confondus dans les critères de choix de l'instrument («Le clarinettiste me paraissait sympathique et le professeur avait une belle moustache, et il parlait avec un accent du Midi, et je me suis dit "tiens, ça c'est un instrument sympathique" ! "Je dirais que je n'arrive plus à déterminer précisément qui de l'un ou de l'autre j'ai choisi. » [Violoncelliste]). 
13 L'instrument se voit aussi prêter des intentions, des caractéristiques ou un caractère humains : la clarinette est un instrument «facétieux » et "mystérieux», le hautbois "pas très virtuose ", le basson a "des personnalités très différentes", "un peu grincheux » ou « un peu goguenard».

14 Mais c'est la comparaison avec la voix humaine et le chant qui signifie en définitive le rapprochement entre l'instrument et l'être humain : l'alto a une sonorité « un peu plus vocale » que le violon, le hautbois est « un instrument assez vocal» dont «le timbre ressemble beaucoup à la voix donc c'est souvent des mélodies très chantées. » En particulier, le violoncelliste reprend ce stéréotype de «l'instrument [...] le plus proche de la voix humaine " spécifique à son instrument en l'élargissant à l'ensemble du registre des voix chantées, "aussi bien la voix du baryton qui chante dans le grave " que celle d'« une colorature, c'est-à-dire une des voix les plus aiguës chez les femmes ", "grâce sans doute à cette tessiture très large de possibilité de la voix humaine, mais aussi à ce son très chaud, très charnel, qui le caractérise. »

La proximité avec l'instrument en fait pour la plupart des instrumentistes un "compagnon» de route, un élément indissociable de leur vie avec qui ils-elles ont établi une relation intime, ainsi que peut le rapporter cet article $^{8}$ de journal relatant la perte de son violon par un violoniste de l'Orchestre de Paris, se sentant «comme un orphelin » et déclarant lors des retrouvailles heureuses suite à une forte mobilisation médiatique : «Mon Lorenzo et moi, ça faisait trente ans qu'on vivait ensemble !»

\subsection{L'identification à l'instrument}

L'identification à l'instrument constitue le pendant de l'humanisation de l'instrument dans la fabrication de l'instrumentiste-instrument. Depuis leur enfance, les instrumentistes ont en effet construit une relation exclusive avec leur instrument : ilselles ont choisi (ou " ont été choisi·e·s par $»^{9}$ ) cet instrument, ont été enseignée's par différente-s enseignant-e· $\mathrm{s}^{10}$ représentant l'instrument, spécifiquement celles et ceux qui sont les plus réputée's au niveau national ${ }^{11}$, travaillé sur des méthodes instrumentales, passé des milliers d'heures à travailler cet instrument seul·e, obtenu des diplômes de/sur cet instrument devant des jurys spécialistes de cet instrument, joué des pièces écrites pour cet instrument, passé pour certainees des concours internationaux dédiés à leur instrument, réussi le concours d'entrée à l'Orchestre de Paris au poste défini par l'instrument, participé à des jurys d'examens ou de concours de cet instrument, sont parfois les partenaires de fabricants ou de luthiers pour la fabrication de cet instrument, membres d'associations qui promeuvent l'instrument et, pour finir, enseignent cet instrument ${ }^{12}$. Ce parcours, spécifiquement fait de rencontres et d'épreuves avec, contre ou pour des instrumentistes qui jouent le même instrument, ressemble à une longue initiation ${ }^{13}$ pour intégrer le groupe des instrumentistesinstruments du même "clan", l'instrument représentant ici « l'espèce de choses qui sert à désigner collectivement le clan [et qui] s'appelle son totem » (Durkheim, 1912b, 108). L'instrument, en tant que totem, "est, d'abord et avant tout, un nom " (Durkheim, 1912b, 115) et contribue à identifier l'instrumentiste. Ainsi, dans l'orchestre, une instrumentiste peut être désignée par le nom même de son instrument : une instrumentiste jouant du basson peut être appelée «basson » au lieu de «bassoniste ». C'est le cas à plusieurs reprises dans le jeu interactif de "Figures de notes » dont sont détaillés quelques exemples ci-dessous : 
17 Un·e flûtiste appelé·e « flûte » :

Image 1. « Jeu Retrouve la flûte»

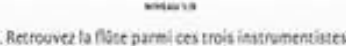
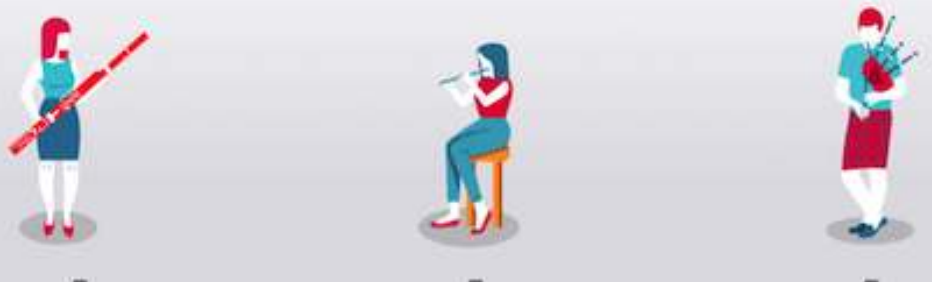

a

18 Un·e hautboïste appelé·e « hautbois »:

Image 2. " Jeu Hautboïste »

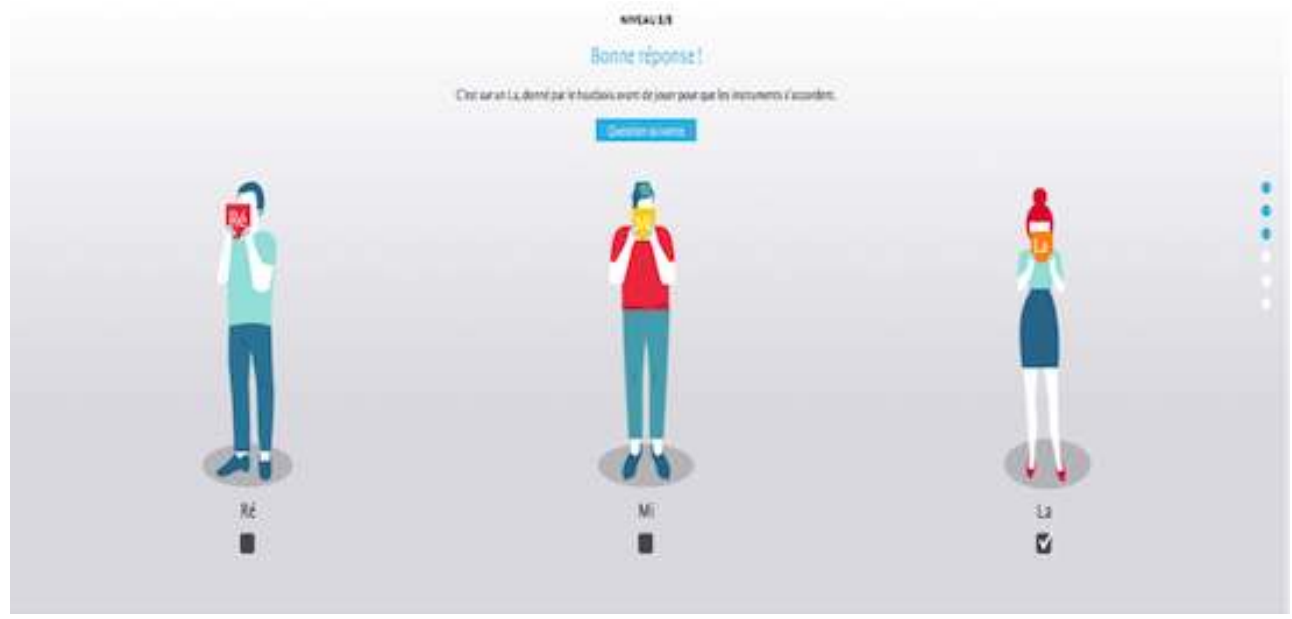

Un·e instrumentiste appelé'e « instrument » : 

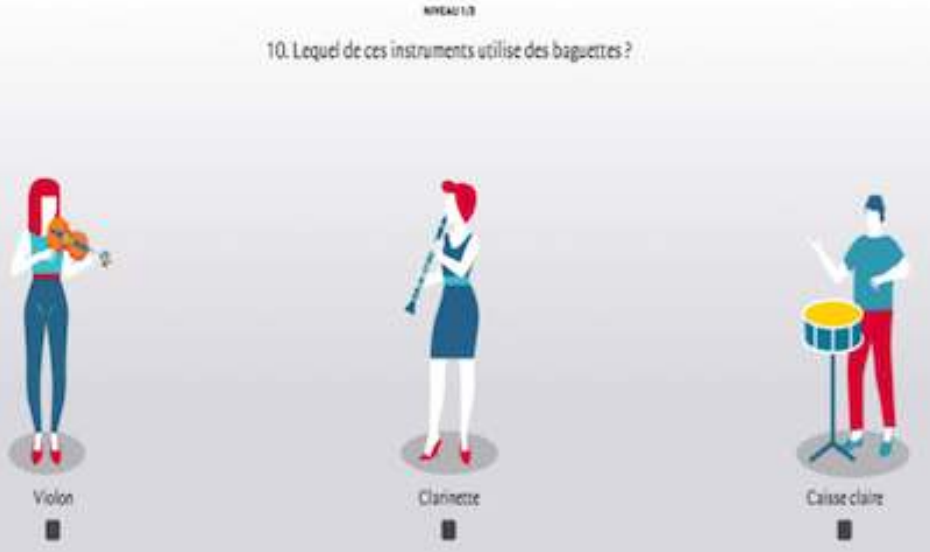

L'utilisation régulière du nom de l'instrument pour l'instrumentiste dans le langage courant devient systématique quand il s'agit d'identifier professionnellement une musicien'ne d'orchestre. L'emploi d'un'e musicien'ne d'orchestre est en effet défini par un poste à tenir au sein de l'orchestre, lui-même défini par la fonction musicale de l'instrument dans l'ensemble des parties de tous les instruments. Par exemple, le poste « clarinette solo » désigne un'e clarinettiste qui a pour rôle d'interpréter les parties de clarinette comportant l'indication «solo » sur la partition du chef d'orchestre appelée " conducteur». La dénomination par le nom et la fonction de l'instrument dans la partition détermine le statut juridique du poste qu'occupe l'instrumentiste. L'instrumentiste n'est pas désignéee par son instrument seul, mais par l'instrument associé à sa fonction (alto du rang, deuxième trompette, etc.).

Cette fonction légale impose ainsi l'utilisation de l'instrument pour l'instrumentiste dans tous les documents administratifs, comme dans l'accord collectif du personnel artistique qui détermine les droits des musicien'ne- $\mathrm{s}^{14}$, et dans la majorité ${ }^{15}$ des annonces de recrutement rédigées en ces termes :

\section{L'Orchestre XXX recrute :}

- une clarinette solo

- deux violoncelles du rang

- une troisième contrebasse solo jouant la contrebasse 5 cordes

- un cor grave jouant le quatrième et le deuxième (3ème catégorie)

L'identification de l'instrumentiste à l'instrument et l'humanisation de l'instrument, par la confusion et l'incorporation qu'elles produisent, contribuent ensemble à la construction de l'instrumentiste-instrument. Ces instrumentistes-instruments ont une place particulière attribuée dans l'orchestre dont les caractéristiques sont observables à travers le dispositif de présentation de « Figures de notes ».

\section{La place de l'instrumentiste-instrument}

«Le rêve, pour un contrebassiste, Schubert... Mais je suis loin du compte, très loin. Je ne suis qu'un tuttiste. C'est-à-dire que ma place est au troisième pupitre. Au premier pupitre, il y a notre soliste et le second soliste ; au deuxième pupitre, le 
chef d'attaque et son second ; et ensuite viennent les tuttistes. Cela n'a pas grandchose à voir avec la qualité, ce sont des emplois codifiés, voilà tout. » (Süskind, 1989, 41).

Dans chaque vidéo, l'installation physique de l'instrumentiste-instrument est la même qu'au sein de l'orchestre ${ }^{16}$. "Figures de notes» signale ainsi que la présentation individuelle de chaque instrumentiste-instrument le-la situe bien collectivement à l'intérieur de l'orchestre, relativement aux autres instrumentistes-instruments. De la même manière, l'analyse de l'ordre et du contenu des 17 vidéos permet de rendre compte de l'attribution d'un placement spatial, d'un rôle musical et d'un statut social spécifiques à chaque instrumentiste-instrument.

\subsection{Un placement spatial}

25 Le plan de présentation des vidéos suit le placement physique des instruments dans l'orchestre, classés et placés en 4 grandes familles : les cordes, les bois, les cuivres et les percussions (dans l'ordre du plus près au plus éloigné du chef d'orchestre).

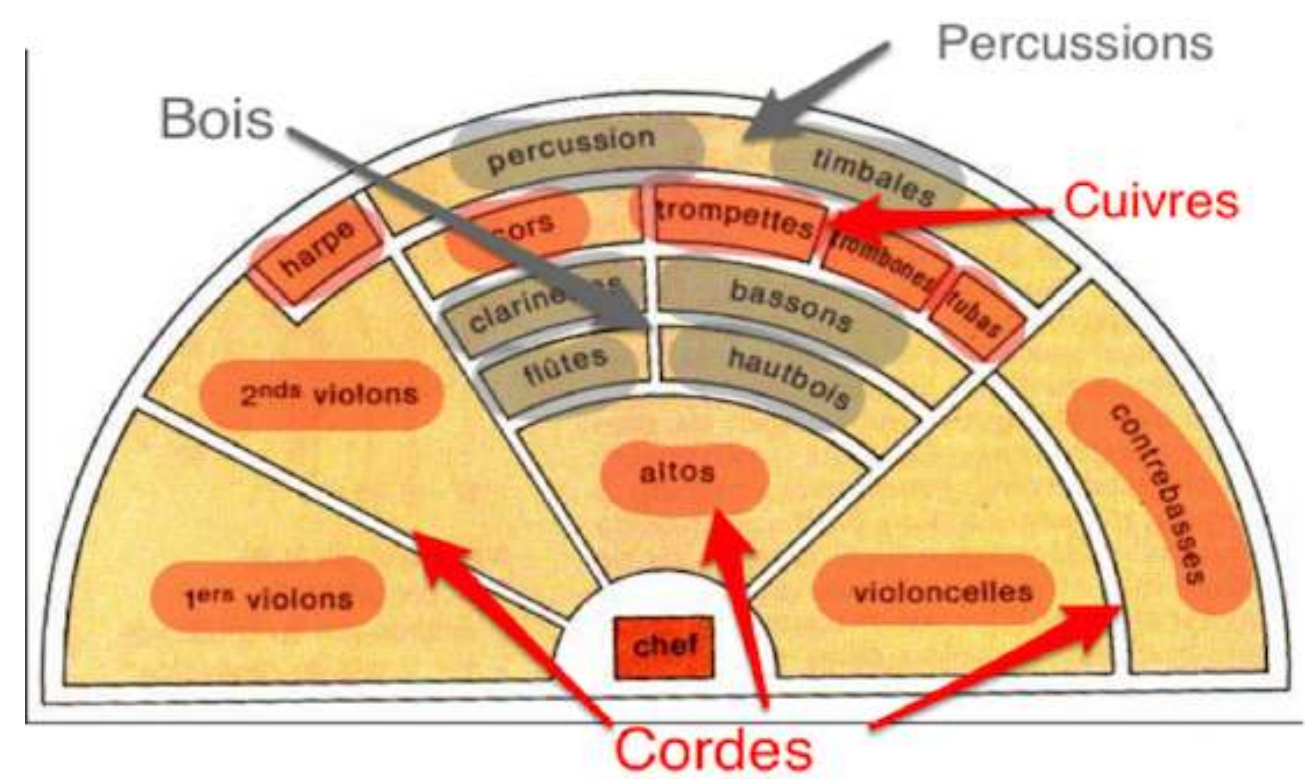

Plan d'orchestre'77 (annoté par l'auteur de l'article)

S'il existe plusieurs dispositions possibles des orchestres symphoniques (Merlin, 2012, 375-376), elles varient assez peu les unes des autres et gardent toutes ce même ordre des familles par rapport au chef d'orchestre. «Figures de notes» reprend cette disposition spatiale par famille pour présenter les deux manières d'accéder aux vidéos des instrumentistes-instruments de l'orchestre (un mur de vidéos et un schéma animé interactif de l'orchestre).

Le mur de vidéo $^{18}$ présente de manière verticale la vidéo de chaque instrumentiste-instrument à l'intérieur des familles classées de la plus proche à la plus éloignée du chef d'orchestre : 


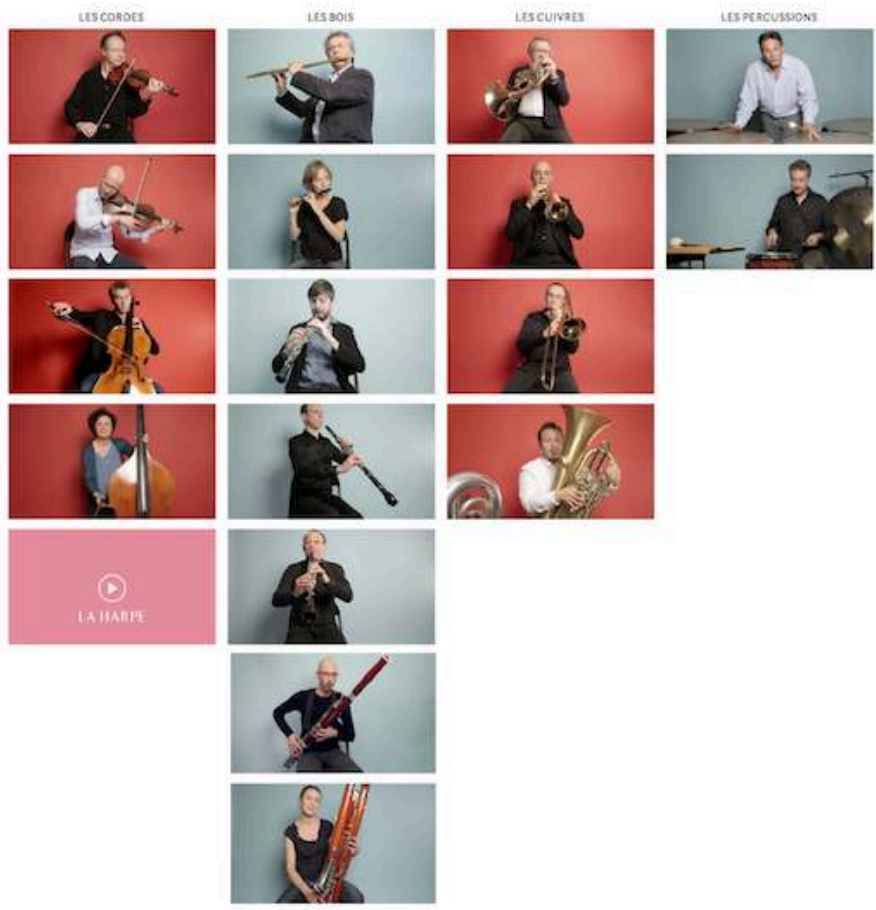

28 À l'intérieur de la famille des cordes $^{19}$, les vidéos suivent un classement de taille croissante d'instrument du plus aigu au plus grave: violon, alto, violoncelle et contrebasse. À l'intérieur de la famille des bois, le classement est déterminé par l'instrument principal d'une nouvelle sous-famille : la flûte, le hautbois, la clarinette et le basson ${ }^{20}$. Pour les cuivres, le cor est placé en premier puis l'on observe un classement par taille et tessiture : trompette, trombone et tuba. Pour la famille des percussions, les timbales précèdent le reste des percussions. Ce classement à l'intérieur de chaque famille correspond à l'ordre dans lequel les instruments sont présentés sur le conducteur qu'utilise le chef d'orchestre, sur lequel sont étagées horizontalement du haut vers le bas les voix correspondant à chaque instrument : 


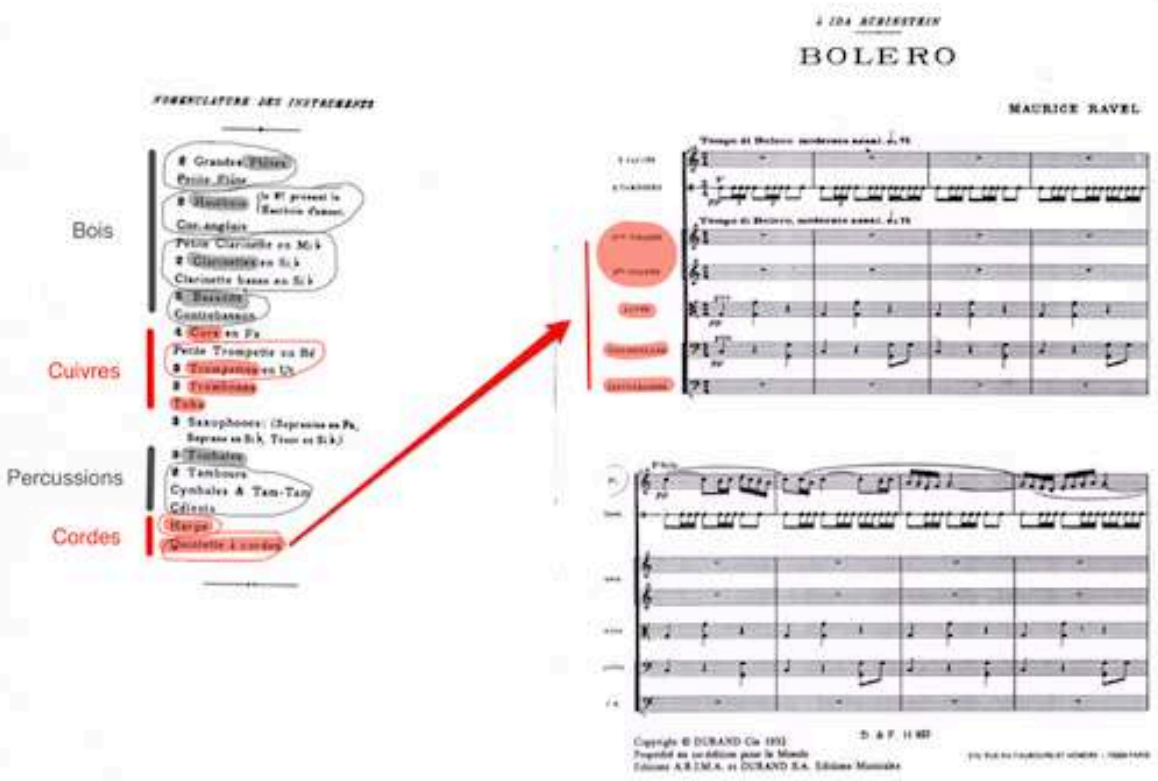

Premières pages du conducteur du « Boléro » de Maurice Ravel (Durand, 1932) (annoté par moi²1)

Si on compare ce conducteur à une carte topographique de l'orchestre envisagée du point de vue du chef d'orchestre, les hiérarchisations haut/bas et près/loin sont respectées pour les bois, les cuivres et les percussions mais pas pour les cordes: positionnées physiquement au plus près du chef d'orchestre, elles sont pourtant tout en bas du conducteur. Cette double place « $\mathrm{au}(\mathrm{x})$ pied(s) du conducteur »- conducteurpartition et conducteur-chef d'orchestre - souligne l'importance structurelle des cordes dans l'orchestre en donnant une image physique de la construction historique de l'orchestre : un ensemble à cordes «sur » lequel ont été ajoutés progressivement les autres instruments (Spitzer et Zaslaw, 2004) ${ }^{22}$.

L'autre accès aux vidéos se fait par une animation interactive du schéma ${ }^{23}$ de l'orchestre vu de dessus. On y retrouve l'emplacement des petits carrés de couleur (rouge ou gris/bleu) qui correspondent à la couleur de fond des vidéos de chaque famille. La vidéo ci-dessous présente l'analyse ${ }^{24}$ de la capture d'écran de cette animation interactive :

Ce média ne peut être affiché ici. Veuillez vous reporter à l'édition en ligne http:// journals.openedition.org/itti/846

Les différentes manières de présenter les instruments sur le site rappellent que le placement physique d'un instrumentiste-instrument obéit à une organisation très précise. Déterminée par le classement des instruments en familles et leur placement physique sur le conducteur du chef d'orchestre, cette organisation spatiale hiérarchique est le reflet d'une organisation musicale elle-même hiérarchique où chaque instrument n'est pas appelé à jouer le même rôle. 


\subsection{Un rôle musical} l'importance des instruments qui jouent ces rôles. Le répertoire des orchestres symphoniques est majoritairement composé d'œuvres tonales pour lesquelles les instruments sont amenés à tenir trois principaux rôles musicaux : un rôle mélodique, un rôle harmonique et un rôle rythmique.

Le «Boléro » de Maurice Ravel, dont chaque instrumentiste joue un extrait au début de sa vidéo, constitue un idéal type de ces trois fonctions ${ }^{25}$. Dans l'extrait du conducteur ci-dessous, le rôle mélodique (thème 1) est attribué au hautbois d'amour (en rouge), le rôle rythmique au tambour et alternativement aux premiers et seconds bassons (en bleu), et le rôle harmonique aux cordes, contrebasses, violoncelles, altos et seconds violons (en vert).

Image 7. «Extrait Boléro »

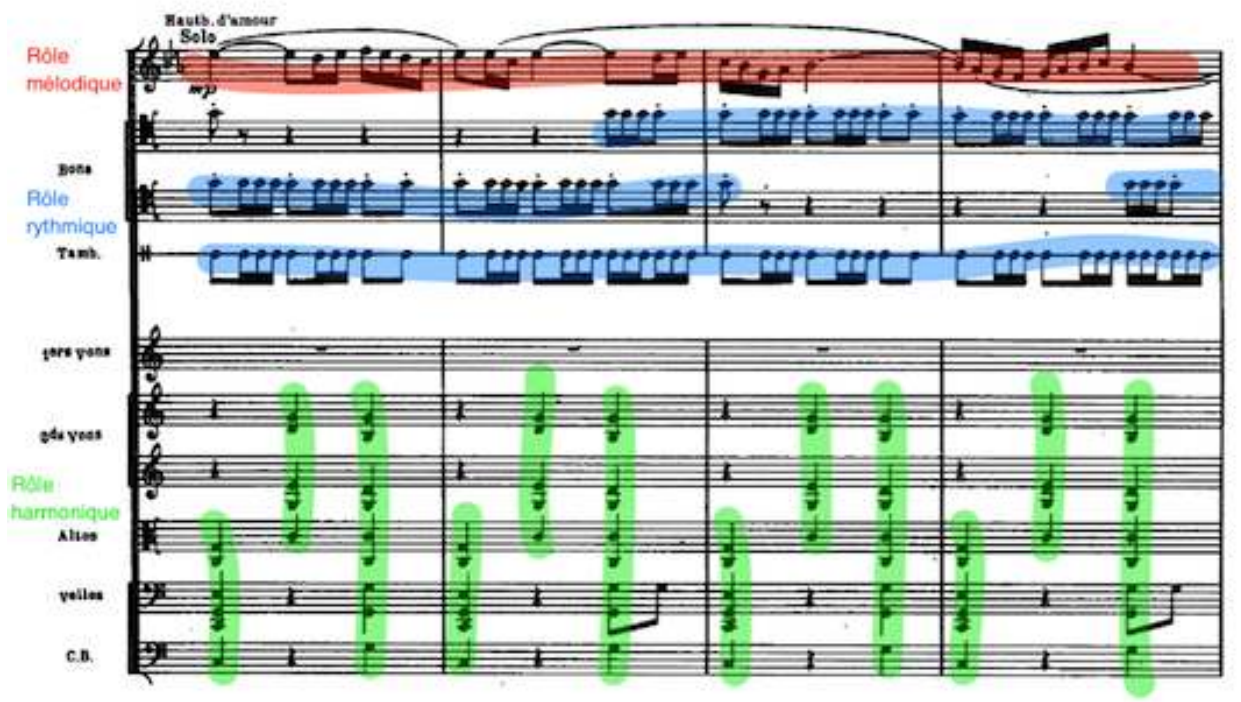

Extrait ${ }^{26}$ du conducteur du « Boléro » de Maurice Ravel (Durand, 1932) (annoté par moi)

Dans l'ensemble du "Boléro", deux thèmes différents de seize mesures chacun (thèmes 1 et 2), joués deux fois de suite à leur tour et séparés de deux mesures à chaque fois, constituent le rôle mélodique. Cette organisation dure pendant tout le morceau, chacun des trois rôles étant réparti successivement entre différents instruments, de plus en plus nombreux pour densifier la masse sonore jusqu'au final. La comparaison des rôles musicaux attribués à chaque instrument par Maurice Ravel avec le rôle musical joué par l'instrumentiste dans la vidéo qui présente son instrument (cf. annexe 2) montre que le rôle mélodique, et plus spécifiquement le thème 1 , est prédominant dans les vidéos ${ }^{27}$. Quatre instrumentistes jouent d'ailleurs le thème 1 alors qu'ils n'ont soit pas de rôle mélodique dans la partition (la contrebassoniste et le tubiste), soit pas ce thème à jouer (le violoncelliste et le bassoniste). Une reconstitution de l'exposition des différents rôles du «Boléro» joués par l'ensemble des instrumentistes illustre la prédominance du thème 1 : 

instrumentale (1er violon, 4ème cor, etc.) en rapport avec les rôles musicaux joués. Ces postes sont répartis en 4 catégories salariales distinctes ${ }^{29}$. L'Orchestre de Paris comporte 26 postes de catégorie 1 (fonction de $1 \mathrm{er}$ solo des instruments principaux ${ }^{30} \mathrm{de}$ chaque famille), 27 de catégorie 2 (fonctions de 2ème solo des instruments principaux de chaque famille + fonction solo des instruments qui constituent une extension ${ }^{31}$ grave ou aiguë de ces instruments), 9 de catégorie 3 (fonction de 3ème solo des instruments principaux de chaque famille) et 55 de catégorie $4^{32}$ (fonction de musicien du rang qui ne concerne que les violonistes, altistes, violoncellistes et contrebassistes tuttistes). Or, les instrumentistes filmé'e-s appartiennent uniquement aux catégories 1 et 2, et ont en très grande majorité une fonction « solo $\|^{33}$. Pour « Figures de notes ", n'importe qui ne peut donc pas présenter un instrument : dans le cas des instruments joués par plusieurs instrumentistes, c'est l'instrumentiste le-la plus hautee dans la hiérarchie musicale et sociale qui présente cet instrument (13 cas sur 17). représentent un tiers de l'effectif total de l'orchestre, les femmes sont sousreprésentées dans les catégories solistes ( $15 \%$ des catégories 1 et 2 ) mais constituent $51 \%$ de la catégorie 4 , musicien'ne du rang ou tuttiste ${ }^{34}$.

À la hiérarchie symbolique liée au rôle mélodique correspond une hiérarchie de pouvoir dans le travail. En effet, les premiers solistes sont également les chefs de pupitre responsables de l'ensemble des instrumentistes qui jouent le même instrument. Les solistes sont les interlocuteurs privilégiés du chef d'orchestre, en haut desquels on 
retrouve le premier violon que les statuts de l'orchestre présentent ainsi : « Le premier violon solo ou l'artiste en assumant la fonction est particulièrement chargé de la discipline artistique au sein de l'Orchestre et, en outre, de la vérification du matériel du quatuor, de l'établissement des coups d'archet suivant les directives du Chef d'Orchestre, des répétitions partielles de pupitre s'il y a lieu et des répétitions du quatuor » (extrait de l'accord collectif du personnel artistique de l'Orchestre de Paris, article 16.3).

Interrogé sur le choix des instrumentistes pour les vidéos, le responsable du site internet a indiqué que, si le choix tenait du volontariat, la demande a d'abord été faite aux premiers solistes par souci de la hiérarchie et parce que ces chefs de pupitre ont l'habitude d'être exposés : «ils sont quand même solistes en permanence dans la vidéo [...] c'est assez périlleux pour eux. »

L'association étroite des rôles musicaux des différents instruments avec les statuts sociaux de leurs instrumentistes détermine le mode de classement et de placement des instrumentistes-instruments dans l'orchestre. Les choix d'organisation de «Figures de notes ", par son titre même ${ }^{35}$, reflètent et confirment «l'enchevêtrement des classifications hiérarchiques entre les instruments et les instrumentistes au sein de l'orchestre" (Adenot, 2008, p. 151): hiérarchies des familles d'instruments, des tessitures, des rôles musicaux, des fonctions, de placement, de sexe, etc. ${ }^{36}$ L'ensemble de ces hiérarchies assigne à chacun'e des instrumentistes-instruments une place à laquelle il-elle semble ajustée, quelle qu'elle soit dans la hiérarchie des places dans l'orchestre. Pourtant, en insistant sur la fonction soliste, le dispositif place certain'e-s des instrumentistes dans une situation de représentation qui ne correspond pas complètement à leur pratique dans l'orchestre et les invite à montrer que leur instrument peut également tenir une autre place.

\section{L'instrumentiste-instrument en représentation}

"Chaque agent a une connaissance pratique, corporelle, de sa position dans l'espace social, un "sense of one's place", comme dit Goffman, un sens de sa place (actuelle et potentielle), converti en un sens du placement qui commande son expérience de la place occupée, définie absolument et surtout relationnellement, comme rang, et les conduites à tenir pour la tenir (« tenir son rang»), et s'y tenir (« rester à sa place », etc.). » (Bourdieu, 2003, 265-266)

En lui demandant de présenter son instrument, le dispositif de « Figure de notes » met l'instrumentiste dans une situation de "présentation de soi " (Goffman, 1973) qui constitue une véritable représentation qui « tend à s'incorporer et à illustrer les valeurs sociales officiellement reconnues, bien plus, en fait, que n'y tend d'ordinaire l'ensemble de son comportement» (Goffman, 1973, 41). Dans la situation dans laquelle est placée l'instrumentiste, seule avec son instrument face à la caméra, la représentation se transforme en représentation de type syndical ou commercial, et l'instrumentisteinstrument devient le-la représentante de l'instrument et de l'ensemble des instrumentistes qui en jouent.

Dans les vidéos, tou'te's les musicien'ne's utilisent à un moment ou à un autre la première personne du pluriel - «nous », «notre » ou le « on » collectif - pour parler de la communauté des praticien-ne's de leur instrument ("Notre répertoire s'étend du XVIIIe au XXIe siècle » [violoniste] ; «Souvent la flûte est utilisée quand il n'y a pas d'accompagnement, on commence nous tous seuls»). Cette utilisation du «nous» 
permet à l'instrumentiste de signaler son appartenance à l'ensemble des instrumentistes qui jouent du même instrument, même pour celles et ceux qui sont les seul'e's à jouer de cet instrument dans l'orchestre, comme la contrebassoniste ou le tubiste. Le « nous » valorise l'instrumentiste par cette appartenance, mais l'oblige aussi à être le-la digne représentante de cet instrument et de cette communauté, et de leur image. Par exemple, si l'alto a été pendant longtemps un instrument joué " par défaut " par des violonistes ${ }^{37}$, les altistes défendent aujourd'hui l'alto comme un instrument « à part entière $»^{38}$ comme le suggère ce qui ressemble pour l'altiste à des excuses publiques: "Je dois avouer que comme beaucoup d'altistes j'ai commencé par le violon. "

Ce "social role", défini comme "l'actualisation de droits et de devoirs attachés à un statut donné» (Goffman, 1973, 24), impose à l'instrumentiste de représenter l'instrument dans toutes ses dimensions. Cela va avoir un effet singulier sur la manière dont l'instrumentiste-instrument va décrire sa place dans l'orchestre: d'un côté, la justification d'être «à sa place » au sein de l'orchestre, d'un autre côté, la nécessité de montrer qu'il-elle peut tenir « une autre place ", à l'intérieur mais également en dehors de l'orchestre.

\section{1. Être à sa place et savoir la distinguer}

Dans les vidéos, les instrumentistes-instruments montrent avant tout qu'ils sont à leur place dans l'orchestre. D'abord en montrant l'expérience en acte de cette place par la connaissance du répertoire et la manière qu'ils-elles ont de le décrire avec précision ; ensuite par le plaisir que leur procure le jeu en orchestre, «le plaisir de partager la musique avec tous », au milieu " de cette puissance sonore, de cette vitalité », qui peut donner « la chair de poule ». Cependant, les instrumentistes ne sont pas "à leur place » de la même façon en fonction de l'instrument joué. Par exemple, il n'y a pas de doute sur la place du violoniste, deuxième violon solo, quand il affirme: "Le violon est omniprésent dans toute la musique orchestrale, tant dans les petites que dans les grandes formations ». La contrebassoniste assume sa place d'une toute autre manière : après avoir présenté son instrument comme n'étant "pas un instrument de soliste ", mais «vraiment un instrument d'orchestre », pleinement consciente du rôle que joue son instrument, elle poursuit ainsi: "J'adore vibrer dans les graves, d'ailleurs j'ai découvert une petite phrase d'un bassoniste qui a vécu ici à Paris, Marius Pierre, qui disait "comprendre l'importance d'une simple note tenue à l'orchestre, c'est participer au même titre que tous à la beauté d'une exécution". Alors faire un simple son dans les graves, une note tenue, ça peut être un plaisir et ça c'est un peu ma mission, le prétexte sous lequel je travaille. »

Valoriser sa place dans l'orchestre demande de valoriser la singularité de cette place dans la hiérarchie des places dans l'orchestre, notamment grâce à un système de distinctions centré sur les caractéristiques de l'instrument. Montrer qu'on est bien à sa place passe ainsi par la comparaison récurrente de son instrument avec les autres instruments. Dans un premier temps, la comparaison sert à pointer la ressemblance, et donc l'appartenance ou la proximité (Contrebassiste: "Il n'y a aucun repère sur le manche, exactement de la même manière que sur le violon, l'alto et le violoncelle "). Mais la comparaison sert également, et souvent dans le même temps, à souligner des différences qui permettent de se démarquer (Clarinettiste : «avec une anche comme le 
basson, comme le saxophone, comme le hautbois, sauf que là c'est une anche simple »). La manière de comparer son instrument à un même instrument permet de montrer une hiérarchie implicite dans l'ordre des instruments : là où le violoniste démarque son violon du piano ( A Au violon, les notes ne sont pas faites toutes seules, contrairement à un piano »), signalant par là un type de supériorité lié au mérite de faire soi-même les notes, le percussionniste, lui, valorise le vibraphone en le rapprochant du piano ( $\mathrm{Ce}$ qui permet de faire des accords courts ou longs, exactement comme au piano »).

Le montage vidéo suivant reprend des extraits où les instrumentistes énoncent des petits « plus » que possède leur instrument par rapport aux autres :

Ce média ne peut être affiché ici. Veuillez vous reporter à l'édition en ligne http://

\subsection{Faire exister et légitimer sa place}

51 Marquer les caractéristiques exclusives d'un instrument est une stratégie pour le faire exister, en particulier parmi ceux d'une même famille, là où les différences sont les moins visibles. Pointer une particularité contribue à s'assurer d'être bien reconnu : le joueur de cor anglais se tient légèrement de profil au début de la vidéo pour qu'on perçoive bien l'angle que fait le bocal de son instrument, caractéristique principale, avec le pavillon en forme de poire, qui distingue hautbois et cor anglais. L'altiste souligne ainsi une habitude de voir confondu son instrument avec un autre :

« Alors une question qu'on me pose très souvent, à l'alto, c'est 'qu'est-ce que c'est ?", notamment quand il est rangé dans sa boite. Ça ressemble à un violon, c'est un peu plus grand, et donc on répond "un alto". Et là, la deuxième question qu'on nous pose en général "ah, un saxophone alto ?" Du coup évidemment, s'ensuivent quelques explications qui permettent, je l'espère, de faire connaître petit à petit l'alto tout court, voilà !»

Pour singulariser leur place, la justifier et la légitimer, les instrumentistes font ensuite référence aux compositeurs. En effet, le compositeur a le pouvoir de faire jouer ou ne pas faire jouer un instrument, de lui attribuer tel ou tel rôle, par l'inscription de notes et d'indications sur la portée du conducteur assignée à l'instrument. Les instrumentistes valorisent ainsi l'instrument par le rôle que les compositeurs lui donnent, citant tel ou tel compositeur qui a écrit des passages intéressants pour leur instrument et insistant même sur les raisons intrinsèques à leur instrument qui ont poussé ce compositeur à l'« utiliser » : « [Le hautbois] est un instrument assez riche en harmoniques, qui a un spectre harmonique très plein donc qu'on entend globalement toujours. Et c'est aussi pour ça que de nombreux compositeurs l'ont utilisé. »

Cependant, le recours à la valorisation par les compositeurs décèle également l'attribution de places symboliques différenciées. Même si l'altiste et le violoncelliste citent tous les deux le poème symphonique «Don Quichotte » de Richard Strauss, dans lequel leur instrument a un rôle soliste, il n'en reste pas moins que le rôle principal, Don Quichotte, est attribué au violoncelliste et celui de Sancho Pança, secondaire, à l'altiste. Dans un autre ordre d'images, le tuba est associé à un rôle d'ours («Petrouchka » I. Stravinski) ou de dragon («Siegfried» R. Wagner) alors que le piccolo est associé à un oiseau («La tourterelle » E. Damaré) et la clarinette à un chat («Pierre et le loup» S. Prokoviev). Et enfin, sans référence à un compositeur précis, la 
description des instruments signale l'habitude de rôles symboliques différenciés : la trompette, « soleil de l'orchestre ", amène " un peu de puissance, d'éclairage " quand la contrebasse permet «d'aller dans les limbes des profondeurs de la musique ».

Les instrumentistes insistent donc sur la place singulière qu'ils ont dans l'orchestre, place déterminée par un ensemble de facteurs liés à leur instrument, lui-même singulier, unique en comparaison aux autres instruments, autant en raison de caractéristiques intrinsèques à la facture instrumentale que d'attribution de rôle musical. En tant qu'instrumentiste-instrument lié à l'orchestre, et malgré les différentes hiérarchies existantes, ils montrent qu'ils sont bien à leur place dans l'orchestre. L'agencement scriptural des différentes parties musicales sur le conducteur et son pendant spatial et sonore dans l'orchestre impliquent cette organisation a priori idéale où chaque instrumentiste est à sa place, c'est-à-dire à la place musicale prévue pour son instrument afin que la combinaison sonore de l'ensemble fonctionne, alternance de temps où le son de l'instrumentiste-instrument se fond dans une masse sonore et de temps où il s'en extrait. Néanmoins, une autre partie de leur discours signale une aspiration à d'autres places pour leur instrument et donc pour eux-mêmes.

\subsection{Vouloir une place en dehors de l'orchestre?}

La plupart des instrumentistes montrent que leur instrument peut faire plus de choses que ce à quoi il est assigné au sein de l'orchestre, suggérant par là qu'une autre place est possible pour eux. Les instrumentistes affectée's plutôt à des rôles musicaux de soutien rythmique et harmonique montrent que leur instrument peut faire non seulement des notes (Timbalier : « les timbales sont un instrument de percussion, c'est un tambour, mais qui ont la particularité de donner des notes") mais également «toutes les notes» (Tubiste: «Mon instrument est pourvu de pistons, ce qui permet d'avoir toutes les notes de la gamme »). En tant que représentant de l'instrument, il est nécessaire de montrer qu'on peut " tout faire ", même avec l'instrument a priori le plus limité : «Le triangle c'est un instrument qui est génial parce qu'on peut faire plein de choses avec, [...] tout est possible. " Il faut entendre ce «tout est possible » comme l'affirmation que l'instrument peut tenir toutes les places, en particulier les plus valorisées: jouer seul le rôle mélodique. Les instrumentistes qui tiennent le plus rarement ce rôle rendent d'ailleurs hommage aux compositeurs qui les mettent « en avant », «en valeur», qui leur permettent d'avoir "leur propre discours » en leur «donnant la parole».

Certaines tournures de phrases font penser qu'au moment de jouer en solo, l'instrumentiste ne fait plus partie de l'orchestre ou, dit autrement, que l'orchestre, c'est les autres : un solo leur permet de "sortir de l'orchestre » (violoncelliste), de se placer «au-dessus de l'orchestre» (joueuse de piccolo), de jouer "au moment où l'orchestre s'arrête » (contrebassoniste), «quand tout le monde s'arrête » (joueur de cor anglais), voire "quand tout le monde est mort» (flûtiste). Les instrumentistes donnent ici une description du soliste qui correspond à celle d'un soliste concertiste qui joue devant, avec ou en dehors de l'orchestre mais qui n'en fait pas partie. L'utilisation du terme soliste pour ces deux places, concertiste et instrumentiste d'orchestre jouant des solos, est donc ambiguë. Le joueur de cor anglais affirme par exemple que " pendant les études musicales, on commence par travailler le hautbois, le répertoire soliste du cor anglais étant quasiment inexistant» mais que «l'utilisation du cor 
anglais en orchestre est plutôt en solo ». Il souligne ainsi que les études musicales visent plutôt le répertoire et la place de concertiste que le répertoire et la place d'instrumentiste d'orchestre, ce qui représente un des reproches récurrents faits par les musicien-ne's d'orchestre à la formation en conservatoire en France (Dupuis, 1993 ; Adenot, 2008).

En quelque sorte, pouvoir justifier du rôle musical mélodique, l'assumer seul et si possible en dehors de l'orchestre, c'est affirmer que son instrument, et donc soi-même, est un individu "à part entière ». Le montage vidéo qui suit montre la manière de décrire l'évolution de l'instrument, des origines «limitées" de rôle rythmique ou harmonique, jusqu'au rôle de soliste qui leur permet de devenir cet instrumentinstrumentiste « à part entière » :

Ce média ne peut être affiché ici. Veuillez vous reporter à l'édition en ligne http:// journals.openedition.org/itti/846

Enfin, certain'e's instrumentistes mettent également en valeur leur instrument dans des pratiques autres que celle de l'orchestre symphonique, mais qui font partie intégrante du répertoire de cet instrument: "La truite» de F. Schubert (contrebassiste) ou les Suites pour violoncelle seul de J.S. Bach (violoncelliste) dans le domaine classique, mais aussi d'autres esthétiques comme le jazz (clarinettiste et percussionniste) ou le funk (tubiste). Si tout cela constitue autant des «clichés » que des pratiques réelles, ces pratiques, culturellement associées à l'instrument, ne peuvent pas ne pas être citées par un'e représentant·e de l'instrument, indiquant ainsi, à côté de celui de concertiste, un autre type d'existence possible de l'instrumentiste-instrument en dehors de l'orchestre ${ }^{39}$.

\section{Conclusion}

« Être musicien professionnel c'est, en effet, aimer son instrument avant toute chose, c'est aimer son instrument avant d'aimer l'œuvre à laquelle on collabore, c'est aimer le toucher, c'est aimer ressentir qu'un trait passe bien, c'est aimer le contact physique et intime que l'on a avec lui. » (Lehmann, 2002)

La place d'un'e musicien'ne d'orchestre est irrémédiablement liée à son instrument. Le dispositif proposé par «Figures de notes » rappelle que le rôle musical attribué à un instrument par le système d'organisation que constitue le conducteur assigne une place spécifique à l'instrumentiste dans l'espace physique et l'espace social de l'orchestre, situant les instrumentistes-instruments dans des positions relatives les unes des autres. Le rapport à cette position est en tension entre deux tendances : d'un côté, comme la place attribuée est pensée et ajustée parfaitement à l'instrument, l'instrumentisteinstrument s'y sent bien, ajustée lui-elle aussi à cette place à l'intérieur d'un réseau de hiérarchies qui structure ces places. De l'autre, comme «dans la plupart des sociétés stratifiées, on idéalise les positions supérieures et on aspire à passer des positions inférieures aux positions supérieures" (Goffman, 1973, 41), les instrumentistesinstruments, dans cette situation de représentation, signalent qu'ils-elles sont capables de tenir une autre place qui apparait plus enviable, « cette attitude ne tradui[sant] pas simplement le désir d'avoir une situation prestigieuse, mais aussi le désir de se rapprocher du foyer sacré des valeurs sociales établies." (Ibid.). En paraphrasant Goffman, on peut dire que la fonction de soliste, dans sa version idéalisée de 
concertiste, constitue le foyer sacré des valeurs musicales établies dans l'orchestre ${ }^{40}$. Tout se passe finalement comme si la place la plus valorisée musicalement et socialement par les musicien'ne's dans l'orchestre - concertiste - était celle qui leur permettrait de se positionner en dehors de cet orchestre.

La notion d'instrumentiste-instrument permet de comprendre cette tension: l'incorporation de l'instrument par l'instrumentiste, fruit d'un long parcours individuel et exclusif avec celui-ci, se transforme en corporation instrumentale qui ne dissocie plus l'instrumentiste de l'instrument. Dans la situation de représentation que constitue ce dispositif, présenter son instrument revient donc à se présenter soi-même, mais également, en tant qu'instrumentiste-instrument, à représenter l'ensemble des instrumentistes de cet instrument, au-delà de la pratique d'orchestre. Parmi l'ensemble des places que peut tenir un instrumentiste-instrument, la place dans l'orchestre, aussi ajustée soit-elle, n'est pas la place symboliquement la plus valorisée dans la communauté de ces instrumentistes-instruments.

\section{BIBLIOGRAPHIE}

Adenot P. (2008), Les musiciens d'orchestre symphonique. De la vocation au désenchantement, Paris, L'Harmattan.

Bourdieu P. (2003), Méditations pascaliennes, Paris, Seuil.

Chagnard S. (2017), « (Re)présentations d'instrumentiste-instrument : variations des stéréotypes de genre en orchestre symphonique. ", in Octobre S. (dir.), Pour des politiques du genre dans le secteur culturel, Paris, ministère de la Culture et de la Communication, Questions de Culture [à paraître].

Coulangeon P. (2004), Les musiciens interprètes en France : portrait d'une profession, Paris, ministère de la Culture et de la Communication, La Documentation française, Département des études et de la prospective.

Dupuis X. (1993), Les musiciens professionnels d'orchestre. Étude d'une profession artistique, Paris, Département des études et de la prospective.

Durkheim E. (1912a), Les formes élémentaires de la vie religieuse. Le système totémique en Australie. Livre I. Questions préliminaires, Édition électronique réalisée par Jean-Marie Tremblay à partir de la cinquième édition, Paris, PUF, 1968, p. 1-138.

Durkheim É. (1912b), Les formes élémentaires de la vie religieuse. Le système totémique en Australie. Livre II. Les croyances élémentaires, Édition électronique réalisée par Jean-Marie Tremblay à partir de la cinquième édition, Paris, PUF, 1968, p. 139-424.

François P. (2009), « La vocation des musiciens : de l'illumination individuelle au processus collectif », Sociologie des groupes professionnels, Paris, La Découverte, « Recherches », p. 165-174 [En ligne] consulté le 13 mars 2016. URL : www.cairn.info/sociologie-des-groupes-professionnels-9782707152145-page-165.htm.

Goffman E. (1973), La mise en scène de la vie quotidienne. 1. La présentation de soi, Paris, Minuit. 
Laillier J. (2017), Entrer dans la danse : l'envers du Ballet de l'Opéra de Paris, Paris, CNRS éditions.

Lehmann B. (2005) [2002], L'orchestre dans tous ses éclats : ethnographie des formations symphoniques, Paris, La Découverte.

Lehmann B. (1995), «L'envers de l'harmonie », Actes de la recherche en sciences sociales, vol. 110, p. 3-21.

Merlin C. (2012), Au cour de l'orchestre, Paris, Fayard.

Papadopoulos K. (2004), Profession musicien « un don », un héritage, un projet ?, Paris, L'Harmattan.

Pégourdie A. (2013), Les provinces de la musique. Pratiques professionnelles, trajectoires et rapports au métier des instrumentistes classiques limougeauds, Thèse de doctorat en sociologie sous la direction de Y. Lamy, Université de Limoges.

Pierre C. (2002), Le Conservatoire national de musique et de déclamation. Documents historiques et administratifs recueillis ou rencontrés par l'auteur, Paris, $C$. Tchou pour la Bibliothèque des introuvables.

Ravet H. (2011), Musiciennes : enquête sur les femmes et la musique, Paris, Autrement.

Reynaud C. (dir.), 2007, L'orchestre de Paris : De la société des concerts du conservatoire à l'orchestre de Paris, 1828-2008, Paris, Centre des monuments nationaux, Éd. du patrimoine.

Spitzer J., et Zaslaw N. (2004), The birth of the orchestra : history of an institution, 1650-1815, Oxford, Oxford University press.

Willener A. (1997), La pyramide symphonique : exécuter, créer ? : une sociologie des instrumentistes d'orchestre, Zürich, Éd. Seismo.

\section{ANNEXES}

\section{Annexe 1 - Matériau utilisé et méthodologie d'enquête}

\section{Matériau :}

- Pages internet de « Figures de notes » :

- 17 vidéos

http://www.orchestredeparis.com/figuresdenotes/index.php ?page =wall-video

- Animation de l'orchestre en hémicycle

http://www.orchestredeparis.com/figuresdenotes/index.php?

page $=$ instrument

- Jeu interactif

http://www.orchestredeparis.com/figuresdenotes/index.php ?page =game

- Entretien avec le responsable du site internet de l'Orchestre de Paris et de «Figures de notes » (par téléphone, le 2/09/15);

- Accord collectif du personnel artistique, statuts et règlement intérieur de l'association Orchestre de Paris ;

- Programme imprimé de la saison 2015/16 ;

- Biographie des 17 musicien'ne's ;

- Conducteur et parties séparées du « Boléro » de Maurice Ravel (Durand, 1932). 
Le matériau premier utilisé pour cette analyse est la retranscription intégrale des 17 vidéos. L'analyse de contenu a consisté à repérer pour chaque instrumentiste les éléments saillants de leur discours, puis transversalement les comparer à l'aune des propriétés, places et rôles musicaux des instruments présentés (par ex. relever systématiquement quels instruments sont cités par quel·le·s instrumentistes et en quels termes, juxtaposer et analyser les réponses aux mêmes questions par famille d'instruments, etc.).

Pour le travail iconographique a été effectuée une analyse du scénario visuel des vidéos, de la mise en forme graphique du mur de vidéos et de la mise en scène des instruments dans l'animation en hémicycle, toujours en lien avec les rôles musicaux des instruments et des statuts sociaux de leurs instrumentistes. Concernant le jeu interactif, après y avoir joué plusieurs fois pour comprendre la réitération des questions et tester des bonnes et mauvaises réponses, j'ai effectué une capture d'écran des 72 questions et 72 réponses proposées pour étudier les termes employés, la représentativité des instruments dans le jeu, etc.

\section{Annexe 2 - Présentation des rôles musicaux du «Boléro »}

\begin{tabular}{|c|c|c|c|c|}
\hline \multirow{2}{*}{$\begin{array}{l}\text { Instrument } \\
\text { joué }\end{array}$} & \multicolumn{3}{|c|}{ Rôles musicaux attribués par Maurice Ravel } & \multirow{2}{*}{$\begin{array}{l}\text { Rôle musical joué dans } \\
\text { la vidéo }\end{array}$} \\
\hline & $\begin{array}{l}\text { Rôle Mélodiq ue (Thème } \\
1 \text { et/ou } 2 \text { ) }\end{array}$ & $\begin{array}{l}\text { Rôle } \\
\text { Harmonique }\end{array}$ & $\begin{array}{l}\text { Rôle } \\
\text { Rythmique }\end{array}$ & \\
\hline Contrebasse & & $\mathrm{x}$ & & Harmonique \\
\hline Timbales & & $\mathrm{x}$ & & Harmonique \\
\hline Alto & 2 & $\mathrm{x}$ & $\mathrm{x}$ & Rythmique \\
\hline Cor & 1 & $\mathrm{x}$ & $\mathrm{x}$ & Rythmique \\
\hline Caisse claire & & & $\mathrm{x}$ & Rythmique \\
\hline Basson & 2 & $\mathrm{x}$ & $\mathrm{x}$ & Thème 1 \\
\hline Clarinette & $1 \& 2$ & $\mathrm{x}$ & $\mathrm{x}$ & Thème 1 \\
\hline Contrebass on & & $\mathrm{x}$ & & Thème 1 \\
\hline Cor anglais & $1 \& 2$ & $\mathrm{x}$ & & Thème 1 \\
\hline Flûte & $1 \& 2$ & $\mathrm{x}$ & $\mathrm{x}$ & Thème 1 \\
\hline Hautbois & $1 \& 2$ & $\mathrm{x}$ & $\mathrm{x}$ & Thème 1 \\
\hline Petite flûte & $1 \& 2$ & & $\mathrm{x}$ & Thème 1 \\
\hline Trompette & $1 \& 2$ & $\mathrm{x}$ & $\mathrm{x}$ & Thème 1 \\
\hline
\end{tabular}




\begin{tabular}{|l|l|l|l|l|}
\hline Tuba & & $\mathrm{x}$ & & Thème 1 \\
\hline Violon & $1 \& 2$ & $\mathrm{x}$ & $\mathrm{x}$ & Thème 1 \\
\hline Violoncelle & 2 & $\mathrm{x}$ & $\mathrm{x}$ & Thème 1 \\
\hline Trombone & 2 & $\mathrm{x}$ & & Thème 2 \\
\hline
\end{tabular}

\section{Annexe 3 - Fonctions et catégories des}

\section{instrumentistes}

\begin{tabular}{|l|l|l|l|l|}
\hline Prénom & Nom & Instrument & Fonction & Catégorie \\
\hline Philippe & Berrod & Clarinette & 1 er solo & 1 \\
\hline André & Cazalet & Cor & 1er solo & 1 \\
\hline Guillau me & Cottet- Dumoulin & Trombone & 1er solo & 1 \\
\hline David & Gaillard & Alto & 1 er solo & 1 \\
\hline Alexand re & Gattet & Hautbois & 1 er solo & 1 \\
\hline Frédéric & Macarez & Timbales & 1 er solo & 1 \\
\hline Éric & Picard & Violoncelle & 1 er solo & 1 \\
\hline Vicens & Prats & Flûte & 1 er solo & 1 \\
\hline Bruno & Tomba & Trompette & 1 er solo & 1 \\
\hline Serge & Pataud & Violon & 2ème solo & 2 \\
\hline Sandrine & Vautrin & Contrebasse & 2 ème solo & 2 \\
\hline Stéphane & Labeyrie & Tuba & solo & 1 \\
\hline Anaïs & Benoit & Petite flûte & solo & 2 \\
\hline Amrei & Liebold & Contrebasso n & solo & 2 \\
\hline Gildas & Prado & Cor anglais & solo & 2 \\
\hline Bord & Martynciow & Percussions & & 2 \\
\hline Nicolas & & & 2 \\
\hline
\end{tabular}




\section{NOTES}

1. http://www.orchestredeparis.com/figuresdenotes/index.php?page=home (consulté le 29/03/16)

2. En parallèle des activités de concert, l'Orchestre de Paris, association subventionnée pour une grande part par l'État et la ville de Paris (à hauteur de 15 M€ en 2008 soit $80 \%$ du budget global), a dans ses missions la mise en place d'actions culturelles en direction de différents publics, en particulier ceux qui ne viennent pas habituellement assister à ses concerts. Une partie de ces actions sont menées par les musicien'ne's.

3. Le matériau et les méthodes utilisés pour l'analyse sont précisés en annexe 1.

4. Titre d'un article sur le site de France Musique : http://www.francemusique.fr/actu-musicale/ la-premiere-harpe-solo-de-l-opera-de-paris-emmaunel-ceysson-integre-le-metropolitan-operade-new-95857 (consulté le 12/01/16).

5. Chacune des 17 vidéos se déroule ainsi : une instrumentiste, positionné·e face caméra sur un fond uni rouge ou bleu clair, présente l'instrument qu'il-elle joue dans l'orchestre en suivant une trame commune, alternant temps de parole et temps de jeu instrumental.

6. Le jeu interactif propose des questions réparties en 3 niveaux de difficulté croissante (10 questions pour le niveau 1, 8 pour le niveau 2 et 6 pour le niveau 3). L'internaute a la possibilité de jouer trois fois de suite au jeu avant d'obtenir à nouveau les mêmes questions : 72 questions sont possibles au total.

7. Dans les vidéos présentées dans cet article, les fondus au noir signalent un montage vidéo effectué par mes soins. Les autres montages sont ceux des vidéos d'origine.

8. http://www.lefigaro.fr/culture/2015/11/05/03004-20151105ARTFIG00065-il-retrouve-sonviolon-rare-apres-l-avoir-oublie-dans-une-autolib.php (consulté le 25/03/16).

9. "Je n'ai pas choisi la contrebasse, on peut dire que c'est plutôt elle qui m'a choisie, car mon premier instrument était la flûte, j'ai commencé la musique comme ça, et c'est mon père qui m'a mis cet instrument dans les mains... »

10. Dont certain'e's par leur père comme la contrebassiste et le trompettiste.

11. 15 des 17 instrumentistes ont fait leurs études dans un des deux Conservatoires Nationaux Supérieurs de Musique et de Danse (CNSMD), 14 à Paris et 1 à Lyon.

12. 3 instrumentistes sur 17 seulement n'enseignent pas. Sur les 14 qui enseignent, 12 le font dans des établissements qui se situent en haut de l'échelle symbolique des établissements d'enseignement en France : 4 au CNSMD de Paris, 1 au CNSMD de Lyon, 6 dans des Conservatoires à Rayonnement Régional de Paris et de sa couronne, 1 à l'École Normale Alfred Cortot et 1 au Pôle d'Enseignement Supérieur de Musique de Bordeaux.

13. «L'initiation est une longue série de cérémonies qui ont pour objet d'introduire le jeune homme à la vie religieuse : il sort, pour la première fois, du monde purement profane où s'est écoulée sa première enfance pour entrer dans le cercle des choses sacrées » (Durkheim, 1912a, 44).

14. À la seule exception du poste concernant le musicien qui joue des timbales, nommé par son nom d'instrumentiste : timbalier. Je n'ai pour l'instant pas trouvé d'explication, au-delà du fait que ce substantif est le seul dans l'orchestre qui ne se termine pas par « iste ».

15. Sauf quelques exceptions comme l'Ensemble intercontemporain, défini comme un « ensemble de solistes ", dont les annonces de recrutement mentionnent le nom de l'instrumentiste : un(e) trompettiste, un(e) clarinettiste, etc.

16. Assise's sur une chaise pour presque tou'te's les instrumentistes, sur un tabouret haut pour la contrebassiste, debout pour le timbalier, et alternativement debout et assis sur un tabouret de batterie pour le percussionniste en fonction des instruments qu'il présente.

17. http://www.icem-freinet.net/ btj/518harmo/plan.jpg (consulté le 10/03/16). 
18. http://www.orchestredeparis.com/figuresdenotes/index.php?page=wall-video (consulté le 19/03/16).

19. Le logo de la vidéo de la harpe est affiché mais la vidéo correspondante n'existe pas.

20. À chacun de ces instruments est associé un instrument qui en représente l'extension vers une tessiture plus aiguë ou plus grave : le piccolo pour la flûte, le cor anglais pour le hautbois et le contrebasson pour le basson. Il manque ici les deux instruments qui sont les extensions de la clarinette, pourtant présents dans l'Orchestre de Paris : la petite clarinette et la clarinette basse.

21. Le saxophone n'est pas présenté dans les vidéos, les saxophonistes n'étant pas permanents dans l'Orchestre de Paris. La portée de la harpe est placée traditionnellement en dessous des percussions sur un conducteur, comme les parties de claviers (piano, célesta, orgue, etc.) quand il y en a.

22. Sur la place spécifique des cordes au sein de l'orchestre d'un point de vue social, voir en particulier les récits des instrumentistes rapportés par Bernard Lehmann (Lehmann, 2005, 167-181).

23. http://www.orchestredeparis.com/figuresdenotes/index.php?page=instrument (consulté le 19/03/16).

24. Les commentaires en sous-titre ont été ajoutés par moi.

25. Comme tout idéal type, ces rôles ne se retrouvent pas de manière aussi nettement séparés dans les œuvres symphoniques.

26. Cet extrait est à écouter entre 3’37 et 3'44 ici: https://www.youtube.com/watch? $\mathrm{v}=8$ po7FZonP-I\#t=3m37s (consulté le 23/03/16).

27. Alors que dans l'ensemble du morceau, le rôle mélodique est dévolu à 12 instruments, le rôle harmonique à 15 instruments et le rôle rythmique à 11 instruments, dans la version filmée, 12 instrumentistes sur 17 jouent la mélodie (dont 11 le thème 1), 2 jouent le rôle harmonique (ici le rôle de la fondamentale de l'accord) et 3 jouent le rôle rythmique.

28. Dans un concerto, une instrumentiste, placée devant l'orchestre à la gauche du chef d'orchestre, tient un rôle mélodique soliste pendant toute l'œuvre. Il s'agit le plus souvent d'un'e musicien.ne invitée et rarement d'un'e musicien'ne de l'orchestre (2 cas sur 25 concertos durant la saison 2015-2016 de l'Orchestre de Paris).

29. Pour donner un aperçu des salaires : en 2001, le traitement de base mensuel brut en début de carrière va de $3113 €$ pour la catégorie 4 à $3871 €$ pour la catégorie 1 . En mai 2017, une annonce de recrutement d'un timbalier solo de catégorie 1 présente un salaire brut mensuel à $4275,34 €$.

30. Violon, alto, violoncelle, contrebasse et harpe pour la famille des cordes; flûte, hautbois, clarinette et basson pour la famille des bois ; cor, trompette, trombone et tuba pour la famille des cuivres ; timbales pour la famille des percussions.

31. Petite flûte, cor anglais, petite clarinette, clarinette basse, contrebasson, trombone basse auxquels s'ajoutent les percussions autres que les timbales.

32. Décompte réalisé à partir de la liste des musiciens de l'orchestre où figurent les fonctions et l'annexe 2 de l'accord collectif du personnel artistique qui définit les catégories salariales à partir des fonctions.

33. Sur les 17 instrumentistes filmé·e·s, 9 sont «premier solo », 2 sont « deuxième solo », 4 sont «solo » et 2 seulement ne sont pas « solo »; 11 sont en catégorie 1, 6 en catégorie 2 mais aucun·e en catégorie 3 ou 4. (cf. Annexe 2).

34. Ces chiffres sont similaires aux autres orchestres symphoniques permanents en France (Ravet, 2011, 200).

35. Dans la théorie de la musique, l'expression «figures de notes » est employée pour définir la hiérarchie des valeurs de durée entre les différentes notes : une ronde est égale à deux blanches, une blanche est égale à deux noires, etc.

36. Auxquelles on pourrait ajouter des hiérarchies liées aux origines sociales des instrumentistes en fonction des instruments joués qui, bien qu'il soit difficile de les observer précisément dans les 
vidéos, sont relevées par les enquêtes sociologiques sur les musicien'ne·s d'orchestre (Lehmann, 2002, 41 ; Ravet, 2011, 293). Une étude plus poussée des stéréotypes de genre à l'œuvre dans le dispositif "Figures de notes", en particulier du jeu interactif, permet de retrouver des différences marquées en fonction du sexe des instrumentistes (Chagnard, 2017).

37. L'alto et le violon sont présents dans l'orchestre depuis le XVIIe siècle (Spitzer \& Zaslaw, 2004) mais, si le violon a été enseigné dès la création du Conservatoire de Paris (1795), l'alto n'y a été enseigné qu'à partir de 1894 (Pierre, 2002).

38. $C f$. paragraphe 3.3 .

39. On retrouve régulièrement la volonté, voire le besoin, de développer d'autres pratiques musicales chez les enquêtée's des études sociologiques sur l'orchestre, que ce soit en musique de chambre (Adenot, 2008, 225 ; Lehmann, 2002, 151), vers d'autres esthétiques musicales (Lehmann, 2002, 160), ou encore d'autres pratiques comme des séances d'enregistrement, la facture instrumentale, etc. (Adenot, 2008, 227-229).

40. Au Ballet de l'Opéra de Paris, univers artistique où les places sont encore plus hiérarchisées, la figure du danseur étoile, dont le statut est « soliste ", joue le même rôle pour les danseurs et les danseuses: "la sanction de l'impossibilité à devenir soliste est perçue comme un refus de reconnaître leur existence. » (Laillier, 2017, 226).

\section{RÉSUMÉS}

L'Orchestre de Paris propose sur son site internet une rubrique "Figures de notes " où 17 musicien'ne-s de l'orchestre présentent leur instrument sous forme de vidéos de dix minutes environ. Cette mise en scène audiovisuelle constitue un matériau iconographique particulier pour saisir les conceptions de ce métier. L'analyse du dispositif audiovisuel de présentation des instruments permet de rendre compte des processus conjoints de personnification de l'instrument et d'identification à l'instrument qui transforment l'instrumentiste en « instrumentiste-instrument ». La place de chaque instrumentiste-instrument est désignée par un placement spatial, un rôle musical et un statut social spécifiques et hiérarchisés. Dans la situation de représentation (Goffman 1973) induite par ce dispositif, le discours des instrumentistes alterne entre l'affirmation et l'appréciation de leur place dans l'orchestre et l'aspiration à une place en dehors de celui-ci, cette tension apparente s'expliquant par la construction même de la figure de l'instrumentiste-instrument.

On its website, the "Orchestre de Paris" shows the section "Figures de notes " where 17 musicians of the orchestra present their instrument in about 10 minutes videos. This mise-en scene is a particular iconographic material to catch significations about musicians' representations. The analysis of the audiovisual device for the instrument's presentation allows to report the process of both personification of the instrument and identification with the instrument that transforms the player into an «instrumentalist-instrument». Each instrumentalist-instrument's place is designated by a spatial placement, a musical role and a social status that are specific and prioritized. In the situation of representation (Goffman 1973), induced by this device, the speech of the instrumentalists alternates between the affirmation and appreciation of their place in the orchestra and the yearning for a place outside of it, the construction itself of the figure of the instrumentalist-instrument explaining this apparent tension. 
INDEX

Keywords : instrumentalist-instrument, symphonic orchestra, representation, musical role, social status

Mots-clés : instrumentiste-instrument, orchestre symphonique, représentation, rôle musical, statut social

\section{AUTEUR}

\section{SAMUEL CHAGNARD}

Musicien multi-instrumentiste, Samuel Chagnard est formateur depuis 2003 au Cefedem Auvergne Rhône-Alpes (Centre de formation des enseignants de la musique). Titulaire du CA de directeur de conservatoire et d'un master en Sciences de l'éducation, il prépare une thèse de sociologie au Centre Max Weber sur la hiérarchisation des pratiques musicales en conservatoire. Site personnel : http://sociomusic.hypotheses.org 